\title{
Research on dynamic receding binary algorithm based on variable slot Hongli Tai ${ }^{a,{ }^{*}}$ and Huan Zhang ${ }^{b}$
}

\author{
Staff University of science and technology of Sichuan, Chengdu 610101, China

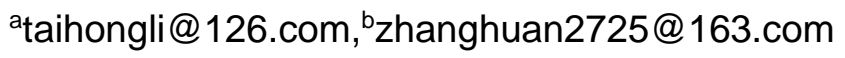

Keywords: Anti-collision, algorithm variable, slot binary algorithm.

\begin{abstract}
This article starts with the anti-collision algorithm of RFID tag. Because of, the recognition rate, recognition speed and channel utilization of the commonly used binary anti-collision algorithm, dynamic frame, slot ALOHA algorithm and dynamic binary tree search algorithm are low, this paper introduces an improved algorithm for dynamic receding binary anti-collision algorithm. Compared with the existing algorithms, the improved algorithm is faster and more efficient, especially in the case of increasing number of tags, the improved algorithm the algorithm has fewer nodes, less time and high efficiency.
\end{abstract}

\section{Introduction}

RFID (Radio Frequency Identification) is a non-contact automatic identification technology, which can acquire the radio frequency signals of objects through electromagnetic induction, and realize data communication and automatic tracking identification with target objects ${ }^{[1]}$. Although RFID technology has been widely used, there are some problems such as security and collision ${ }^{[2]}$. Anti-collision algorithm has become an effective way to improve system identification speed, improve communication reliability, reduce failure rate and error rate, and improve the efficiency of RFID system work.

In the RFID system, when multiple tags respond to different queries reader at the same time, the communication collision between the reader and the tag is caused. Therefore, a collision mechanism is needed to minimize communication collisions between the two. If there is no corresponding collision mechanism, the reader will not be able to read the contents of the tag properly, leading to the identification failure. At present, considering the low cost, low power consumption, low storage capacity and small computation, in all RFID anti-collision methods the TDMA method is widely used. There are two most commonly used TDMA, one is based on statistical ALOHA algorithm, and the other is deterministic binary tree search algorithm ${ }^{[3]}$. ALOHA algorithm transmits data at different times, so that no collision between tags will occur, and the transmission gap of each tag should be long enough. This algorithm is usually applied to low frequency passive read only tags. It has the advantages of low collision rate, simple design and high security, and the drawback is that the channel utilization is low, randomness is large, and the rate of false positives is high. This algorithm has the advantages of low collision rate, simple design and high security, and has the disadvantage of low channel utilization rate, large randomness and high misjudgment rate. By sending commands repeatedly, the labels are divided into two groups by the command sent each time, binary tree search algorithm gets unique labels finally. The advantages of this algorithm are high stability, high channel utilization, strong recognition ability and easy implementation of software. The disadvantage is that the security is low and the time delay is long.

Based on ALOHA algorithm and binary tree search algorithm, many improved algorithms have been proposed. Among them, the improved ALOHA algorithm mainly includes time slot ALOHA algorithm, frame slot ALOHA algorithm, dynamic frame slot ALOHA algorithm and so on. The improved binary tree algorithm mainly includes pruning tree, binary tree search algorithm, dynamic binary tree search algorithm and backward binary tree search algorithm and so on ${ }^{[4]}$. The above two algorithms have their own advantages, and the recognition rate, recognition speed and channel utilization of the two algorithms are low. In addition, the complexity of the identification process 
needs further research and improvement. In view of this, this paper introduces a dynamic backward binary algorithm based on variable slot.

\section{Dynamic Receding Binary Algorithm Based on Variable Slot}

The dynamic receding binary algorithm based on variable slot combines the advantages of the dynamic frame slot algorithm (DFSA) and the dynamic backward binary search algorithm (DRBS) and adds the tag number estimation model (Vogt) to the algorithm. Variable slot anti-collision and dynamic frame slotted ALOHA are algorithms that change the number of slots, increase or decrease the frame length according to the tag collision rate.

The algorithm is the dynamic receding binary search algorithm based on variable slot, which improved on the basis of binary search algorithm(DBSR VTS). All tags are modeled as a two binary tree according to their serial numbers. When a collision occurs, after successfully identified by the algorithm, the next traversal starts with the parent node instead of the root node, avoiding repeated operations. At the same time, the binary search algorithm sends an order equal to the number ID, which asks every time, whereas, the dynamic backward binary improvement algorithm only needs to send Request (x,y) commands at a time ( $\mathrm{x}$ stands for the collision occur at the highest point, and $\mathrm{Y}$ is the assignment of the maximum and sub maximum collision bit).That is, the tag of the next collision node is identified by using the back off strategy by locking the colliding bits in this algorithm,.

\subsection{Algorithm Principle}

First, set the command Set slot (1), Set the current slot number to 1 (label self selection slot).

Second, send Request (null, 8) command, and request the current region label upload its ID number.

Third, according to the collision rate, the number of tags is estimated using Vogt tag number estimation model. At the same time, the optimum slot number $\mathrm{x}$ which in the current system is obtained by using the optimal frame length formula.

Fourth, send the Set slot (x) command again to reset the label slot number.

Fifth, send Request (null, 8), asking the label to upload the ID number according to the slot.

Sixth, identify tags according to the dynamic backward binary search algorithm. A collision occurs during the identification of the tag, There are three kinds of collisions: the tag collision bit exceeds 2 bits, a tag collision bit is equal to a 2 bit situation and tag collision bits in 1 bit situations. When the tag collision bit exceeds 2 bits, $\mathrm{R}(\mathrm{Z}, \mathrm{x}, \mathrm{y})$ instructions are used, in which $\mathrm{Z}$ represents the slot number, $\mathrm{X}$ is the highest hit bit number, and $\mathrm{Y}$ is the highest 2 bit collision bit value; when the tag collision bit is equal to 2 bits, the $\mathrm{E}(\mathrm{Z}, \mathrm{x}, \mathrm{y})$ instructions are used, $\mathrm{Z}$ is the slot number, $\mathrm{X}$ is the highest hit bit number, and $\mathrm{Y}$ is the bit value; When the tag collision bit is 1 , it is directly be identified.

\subsection{Tag Number Estimation Model}

Vogt specifies that the number of empty slots is expressed as $c_{0}^{\prime}$, which read by a reader, when the read success, the slot of number is expressed as $c_{1}^{\prime}$, when the tag collision, the slot number is expressed as $c_{k}^{\prime}$, Accordingly there are vectors $\left(c_{0}^{\prime}, c_{1}^{\prime}, c_{k}^{\prime}\right)$, theoretical empty slot numbers $\alpha_{o}^{L, t}$, the successful slot number $\alpha_{1}^{L, t}$, the collision slot number $\alpha_{k}^{L, t}$, and they consist of vectors $\left(\alpha_{o}^{L, t}, \alpha_{1}^{L, t}\right.$, $\left.\alpha_{k}^{L, t}\right)$.The two sets of vector distances are $\varepsilon_{v d}$, select the smallest $\mathrm{t}$ in $\varepsilon_{v d}$ as the tag to estimate the number, and use the following formula:

$$
\begin{aligned}
& \varepsilon_{v d}\left(t, c_{0}^{\prime}, c_{1}^{\prime}, c_{k}^{\prime}\right)=\min _{t}\left\|\left(\begin{array}{l}
\alpha_{o}^{L, t} \\
\alpha_{1}^{L, t} \\
\alpha_{k}^{L, t}
\end{array}\right)-\left(\begin{array}{l}
c_{0}^{\prime} \\
c_{1}^{\prime} \\
c_{k}^{\prime}
\end{array}\right)\right\| \\
& \alpha_{r}^{L, t}=L\left(\begin{array}{l}
t \\
r
\end{array}\right)\left(\frac{1}{L}\right)^{r}\left(1-\frac{1}{L}\right)^{t-r}
\end{aligned}
$$

Among them, $\mathrm{t}$ is the estimate of the number of tags. 


\subsection{Simulation and Analysis}

The simulation selects 200 bit electronic tags, the simulation data is run 10 times in each group, and the average result of 10 runs is plotted as follows:

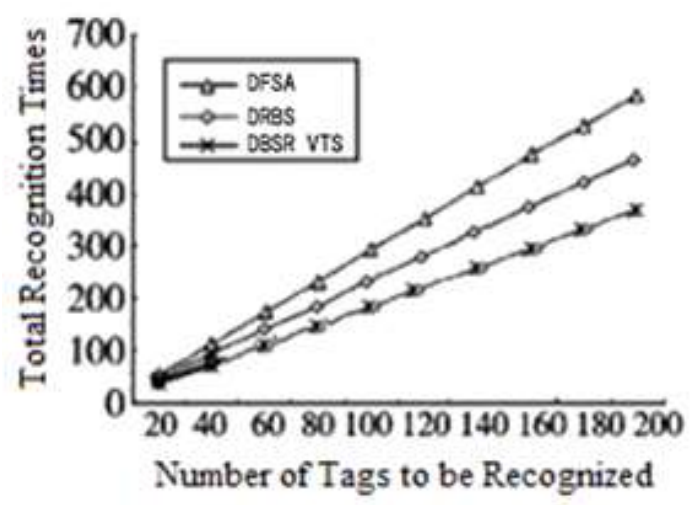

Fig. 1 Comparison of Recognition Times

Figure 1 shows that the new algorithm requires less total number and shorter recognition time in the case of identifying the same multiple tags. When the total number of tags to be recognized is 200 , the total number of recognition times of the new algorithm is less than $21 \%$ of the DRBS, and less than $36 \%$ of the DFSA. Thus, the more the number of tags to be identified, the better the new algorithm is.

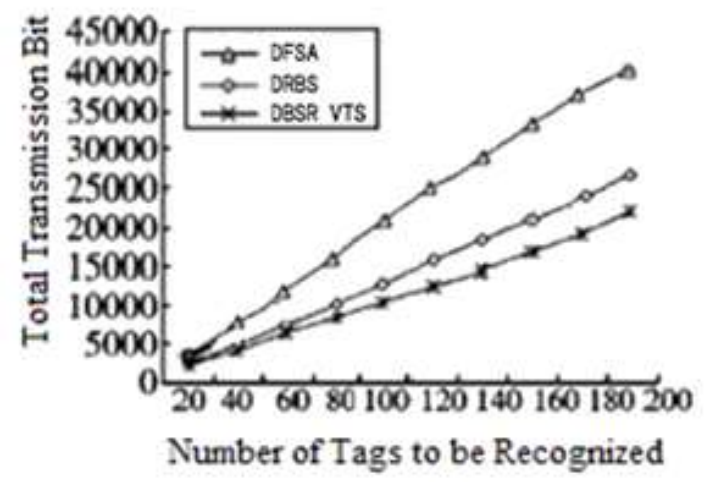

Fig. 2 Transmission Data Volume Comparison

As shown in Figure 2, when the number of tags is 200, the new algorithm identifies less than $29 \%$ of the total number of bits transmitted, and less than $37 \%$ of the DFSA.

Therefore, the new algorithm can reduce the collision, the total number of recognition, and the total amount of data transmitted, which introduced in this paper.

\section{Conclusion}

From the above reasoning and analysis, it can be seen that the improved algorithm introduced in this paper is faster, more efficient, especially in the case of increasing number of tags, the dynamic receding binary algorithm based on variable slot traverses the nodes with fewer operating tags, less time, and higher traversal efficiency.

\section{References}

[1]. R. Want, An introduction to RFID technology. IEEE Pervasive Computing. vo1.5, No.1, PP.2533, Jan.2006.

[2]. Z.Q.You. Radio frequency identification (RFID) theory and Application Publishing House of Electronics Industry, Bei Jing, 2004.

[3]. X.Chen, X.P.Zhang and S.D.Zhang. Research on Anti-collision Algorithm of tag J. Modern Electronics Technique, 2006,(5):13-15.

[4]. S.R.Nie and Y.YLiu. A survey of current research situation and development trend of RFID anticollision algorithm J. Logistics Technology, 2008,(03):75-78. 